\title{
Cytogenetics and morphology of two tortoise species of the genus Chelonoidis (Fitzinger, 1835) (Testudines)
}

\author{
T.L. Silva
}

2011. Universidade Estadual Paulista "Júlio de Mesquita Filho", São José do Rio Preto, SP, Brasil. MSc. thesis. Orienting Prof.: Maria Tercília Vilela de Azeredo Oliveira. Co-orienting Prof.: Claudia Regina Bonini Domingos.

DOI 10.4238/vol10-2ta034

The reptiles were reduced in number of species since the time that they ruled the earth until today. The tortoises have been poorly studied, particularly their morphological and cytogenetic features. We examined the species Chelonoidis carbonaria and C. denticulata, terrestrial tortoises representative of two contrasting biomes (Cerrado and Amazonia), to check for the existence of a possible $C$. carbonaria morphotype, and describe their karyotypes. The animals were collected from the "Reginaldo Uvo Leone" breeding farm, located in Tabapuã, SP. We made measurements of external morphology, in order to evaluate the morphological characteristics that could effectively allow differentiation of these two species of Brazilian tortoises, also examining a C. carbonaria morphotype that differs in color and size patterns established for this species. This C. carbonaria morphotype showed morphological characteristics intermediate between these two species described. Cytogenetic studies gave a chromosome number of $2 n=52$ for all three groups. G-banding did not give good reproducibility and consistency in the banding patterns. In C. carbonaria males, C-banding technique revealed constitutive heterochromatin in two microchromosomes and in two centromeric regions of macrochromosomes; in females only two microchromosomes showed specific staining. The species $C$. denticulata and the $C$. carbonaria morphotype did not have evident heterochromatic blocks. Silver staining technique (Ag-NOR) revealed markings in the 10th pair of chromosomes in all the tortoises. Males had telomeric regions of an acrocentric macrochromosome pair; females had only a single acrocentric macrochromosome of the same pair. In situ hybridization with an amphibian 28S rDNA probe showed two NORs in males and females of the three groups of tortoises. Based on the morphological data, we suggest that the $C$. carbonaria morphotype corresponds to a new tortoise species; however, a broader evaluation of morphological, genetic and geographic data will be necessary in order to assess the conservation status and the occurrence area of this morphotype. These findings contribute to our knowledge of tortoise biology and evolution, generating data that could contribute to the preservation of this important group of reptiles.

Key words: Chelonoidis carbonaria; C. denticulata; Morphotype; Cytogenetics; Tortoises 